\title{
Anomalous Interaction of Longitudinal Electric Field with Hydrogenated Amorphous Silicon Films
}

\author{
Jingyu Zhang ${ }^{1 *}$, Mindaugas Gecevičius ${ }^{1}$, Martynas Beresna ${ }^{1}$, Andrey G. Kazanskii ${ }^{2}$, Peter G. Kazansky ${ }^{1}$ \\ ${ }^{1}$ Optoelectronics Research Centre, University of Southampton, SO17 1BJ, United Kingdom \\ ${ }^{2}$ Physics Department, M.V. Lomonosov Moscow State University, Moscow, Russia \\ jz2e11@orc.soton.ac.uk
}

\begin{abstract}
Cylindrically polarized beams produced by femtosecond laser written S-waveplate are used to modify amorphous silicon films. Paradoxically, no crystallization is observed in the maximum of longitudinal electric field despite the strongest light intensity.
\end{abstract}

OCIS codes: (140.3390) Laser materials processing; (260.7120) Ultrafast phenomena; (160.2750) Glass and other amorphous materials; (260.5430) Polarization

A strong longitudinal electric fields at the focal region has raised great interest in the laser fabrication and microscopy [1,2]. At the focus radially polarized annular ring can be decomposed into longitudinally and transversely polarized electric fields [1]. The transverse part of the electric field forms a ring, while longitudinal in concentrated in the centre. Due to longitudinal component the spot size of the beam can be smaller than predicted by diffraction limit. Here we demonstrate that thin film of amorphous silicon exhibits anomalous polarization selective absorption in case of radially polarized beams.

The experiments were performed with femtosecond laser system Pharos (Light Conversion Ltd.) based on a directly diode pumped $\mathrm{Yb}: \mathrm{KGW}$ crystal, operating at $\lambda=1030 \mathrm{~nm}$ and delivering pulses of $270 \mathrm{fs}$ at $20 \mathrm{kHz}$ repetition rate. Laser beam was focused with a $1.2 \mathrm{NA}$ water immersion lens on the surface of hydrogenated amorphous silicon thin film mounted on a three-axial motion platform. The radial polarization was obtained by inserting a laser written S-waveplate into the path of linearly polarized laser beam just before the objective [3]. The annular beam was generated by modulating beam's phase with liquid crystal spatial light modulator.
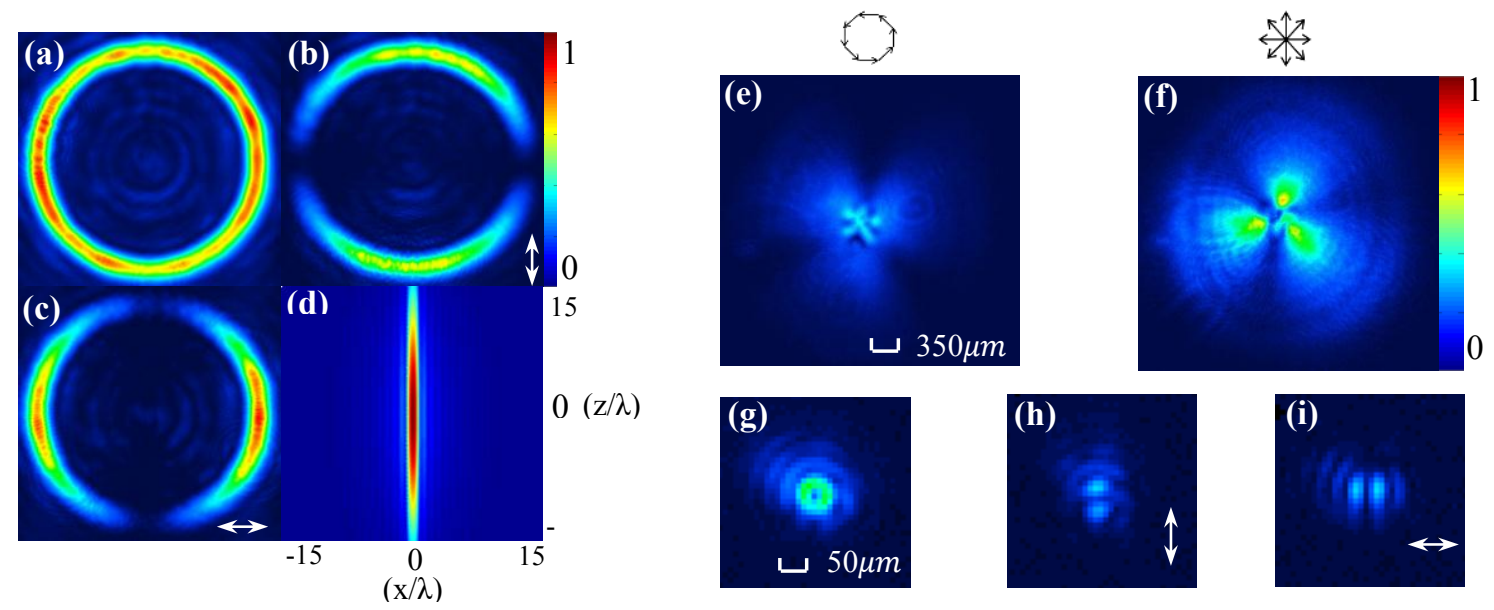

Fig. 1. (a)-(c) are the normalized intensity distributions measured by the CCD camera: (a) the radial ring-shape beam. (b) and (c) is the intensity distributions through linear polarizers. (d) Intensity distribution in the xz plane when the radially polarized beam is focused with a

1.2 NA water immersion objective lens. (e)-(i) are intensity distributions of second harmonic generated through lithium niobate.(e)-(f) Second harmonic signal generated in lithium niobate for radially, azimuthally polarized Gaussian profile in the far field measured by the CCD camera. (g) Second harmonic signal for radially polarized Gaussian profile in the near field. (h) and (i) is the intensity distribution through linear polarizers. The arrows indicate the transmission axis of the polarizer.

The modelling revealed that the tightly focused ring-shaped radially polarized beam should create a strong longitudinal electric field, whose intensity is twice as high as the transverse component with a long depth of focus $(\sim 24 \lambda)$ (Fig. 1). However, the highest intensity of the transverse component of the annular azimuthally polarized beam is $65 \%$ higher than the radially polarized beam. The presence of longitudinal component was confirmed by second harmonic generation in z-cut lithium niobate wafer. The second harmonic was generated by focusing annular 
radially polarized femtosecond laser beam with a $1.2 \mathrm{NA}$ water immersion microscope objective. The produced second harmonic was collected with high NA objective after the sample. We observed twice higher second harmonic power for the radially polarized annular beam compared with the azimuthally polarized one corresponding to the contributions of first harmonic longitudinal field towards the transverse field of the second harmonic. In this case, the gain of second harmonic signal is obtained due to the longitudinal component. In the near field, the second harmonic formed a ring which was radially polarized. In the far-field second harmonic signal was in shape of threelobes for radial and azimuthal polarizations (Fig.1 (e)-(f)) indicating spatially variant phase of the beam. Moreover, the pattern of the second harmonic was rotating while rotating the sample, thus it depends on the orientation of lithium niobate.

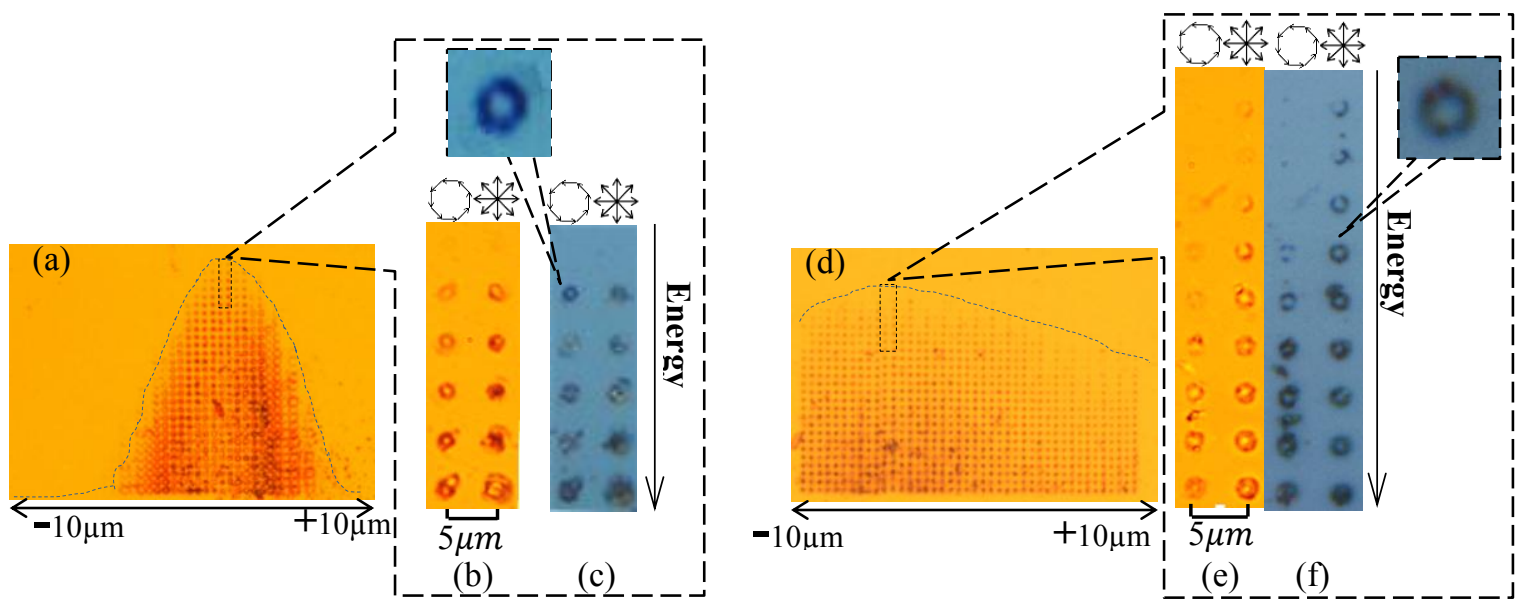

Fig. 2. Microscope image of the structures on amorphous silicon by Gaussian and ring-shaped radially and azimuthally polarized beams. (ac) Sequences of structures modified by Gaussian radially and azimuthally polarized beams at different focus plane. (d-f) Sequences of structures modified by ring-shaped radially and azimuthally polarized beams at different focus plane. Microscope images from transmission $(\mathrm{b}, \mathrm{e})$ and reflection (c, f) view respectively.

The annular radially polarized beam was used to modify $300 \mathrm{~nm}$ thick hydrogenated amorphous silicon films [4]. In the experiment 20 sequences of dots were printed by irradiating single radially or azimuthally polarized pulses at different focusing depth ranging from $-10 \mu \mathrm{m}$ to $10 \mu \mathrm{m}$. The energy was varied from 0 to $75 \mathrm{~nJ}$ for beam with Gaussian profile and 0 to $300 \mathrm{~nJ}$ for beam with annular-ring shape. The dots were separated by $5 \mu \mathrm{m}$. After irradiation the samples were inspected with an optical microscope. For Gaussian radially polarized beam the crystallization of the amorphous silicon started at the same energy for radial and azimuthal polarizations. The modification threshold strongly depended on the focusing conditions due to short Rayleigh range of the beam. On the contrary, weak dependence on focusing was observed for annular laser beam indicating Bessel-like long Rayleigh range. With annular beam the difference between radial and azimuthal polarizations was very strong. The threshold for permanent modification threshold was more than $40 \%$ lower for radial polarization than for azimuthal. This indicates larger fluence of the radially polarized annular beam, which should be related to the enhancement of longitudinal polarization. However, in both cases the ring shape of laser printed dots was observed. The middle of the structure, where strong longitudinal electric field was present, was not modified. This contradicts common notion that strength of modification should be dependent on the fluence of the laser beam. We speculate that this curious effect could be related to coupling of electric field into surface waves which redistributes energy over large area. The detailed mechanism of longitudinal polarization interaction at the interface of the thin film is currently under investigation.

\section{References}

[1] R. Dorn et al, "Sharper focus for a radially polarized light beam," Phys. Rev. Lett. 91, 233901 (2003).

[2] C. Hnatovsky et al. "Revealing local field structure of focused ultrashort pulses," Phys. Rev. Lett. 106, 123901 (2011).

[3] M. Beresna et al. "Radially polarized optical vortex converter created by femtosecond laser nanostructuring of glass," Appl. Phys. Lett. 98, 201101 (2011).

[4] A. V. Emelyanov et al. "Visible luminescence from hydrogenated amorphous silicon modified by femtosecond laser irradiation," Appl. Phys. Lett. 101, 081902 (2012). 